OPEN ACCESS

Edited by:

Akio Adachi,

University of Tokushima, Japan

Reviewed by:

Daniel C. Pevear,

VenatoRx Pharmaceuticals, USA

Chuan Xiao,

University of Texas at El Paso, USA

Sindy Böttcher

Robert Koch Institute, Germany

${ }^{*}$ Correspondence: Maël Bessaud mael.bessaud@pasteur.fr

Specialty section: This article was submitted to Virology,

a section of the journa

Frontiers in Microbiology

Received: 26 May 2016 Accepted: 05 August 2016

Published: 26 August 2016

Citation

Bessaud M, Sadeuh-Mba SA, Joffret $M-L$, Razafindratsimandresy $R$ Polston P, Volle $R$, Rakoto-Andrianarivelo $M$, Blondel $B$ Njouom R and Delpeyroux F (2016) Whole Genome Sequencing of Enterovirus species $C$ Isolates by High-Throughput Sequencing: Development of Generic Primers.

Front. Microbiol. 7:1294.

doi: 10.3389/fmicb.2016.01294

\section{Whole Genome Sequencing of Enterovirus species $C$ Isolates by High-Throughput Sequencing: Development of Generic Primers}

\author{
Maël Bessaud ${ }^{1,2,3 *}$, Serge A. Sadeuh-Mba ${ }^{4}$, Marie-Line Joffret ${ }^{1,2,3}$, \\ Richter Razafindratsimandresy ${ }^{5}$, Patsy Polston ${ }^{1,2}$, Romain Volle ${ }^{1,2}$, \\ Mala Rakoto-Andrianarivelo ${ }^{6}$, Bruno Blondel ${ }^{1,2}$, Richard Njouom ${ }^{4}$ and \\ Francis Delpeyroux ${ }^{1,2,3}$
}

${ }^{1}$ Unité de Biologie des Virus Entériques, Institut Pasteur, Paris, France, ${ }^{2}$ Institut National de la Santé et de la Recherche Médicale, U994, Paris, France, ${ }^{3}$ WHO Collaborating Center for Research on Enteroviruses and Viral Vaccines, Institut Pasteur, Paris, France, ${ }^{4}$ Centre Pasteur du Cameroun, Service de Virologie, Yaoundé, Cameroon, ${ }^{5}$ Unité de virologie, Institut Pasteur de Madagascar, Antananarivo, Madagascar, ${ }^{6}$ Centre d'infectiologie Charles-Mérieux, Université Ankatso,

Antananarivo, Madagascar

Enteroviruses are among the most common viruses infecting humans and can cause diverse clinical syndromes ranging from minor febrile illness to severe and potentially fatal diseases. Enterovirus species $C$ (EV-C) consists of more than 20 types, among which the three serotypes of polioviruses, the etiological agents of poliomyelitis, are included. Biodiversity and evolution of EV-C genomes are shaped by frequent recombination events. Therefore, identification and characterization of circulating EV-C strains require the sequencing of different genomic regions. A simple method was developed to quickly sequence the entire genome of EV-C isolates. Four overlapping fragments were produced separately by RT-PCR performed with generic primers. The four amplicons were then pooled and purified prior to being sequenced by a high-throughput technique. The method was assessed on a panel of EV-Cs belonging to a wide-range of types. It can be used to determine full-length genome sequences through de novo assembly of thousands of reads. It was also able to discriminate reads from closely related viruses in mixtures. By decreasing the workload compared to classical Sanger-based techniques, this method will serve as a precious tool for sequencing large panels of EV-Cs isolated in cell cultures during environmental surveillance or from patients, including vaccine-derived polioviruses.

Keywords: Enterovirus species C, poliovirus, high-throughput sequencing, recombination, de novo assembly

\section{INTRODUCTION}

The members of the Enterovirus species C (EV-C), genus Enterovirus, family Picornaviridae, are non-enveloped viruses with a single positive strand RNA genome. The virions contain one copy of the genome, which is about 7500 nucleotides in length and consists of two untranslated regions ( $5^{\prime}$ - and $3^{\prime}$-UTR) flanking a unique large open reading frame. The polyprotein encoded by this open reading frame is first cleaved into three precursors (P1-P3) that are subsequently cleaved into 
functional proteins. P1 gives rise to the four structural capsid proteins (VP1-VP4) while P2 and P3 generate non-structural proteins involved in the viral cycle. Enteroviruses cause a wide spectrum of human diseases, with clinical signs ranging from mild febrile illness, such as the common cold, to severe forms, such as acute haemorrhagic conjunctivitis, myocarditis, encephalitis, and acute flaccid paralysis (Tapparel et al., 2013).

Currently, more than 20 types of EV-Cs have been identified (Knowles et al., 2012), including the three serotypes of poliovirus (PV-1 to -3$)$ that can induce severe and potentially fatal cases of poliomyelitis in humans. Typing of enteroviruses relies on molecular characterization of the capsid-encoding region (Blomqvist and Roivainen, 2016). For this purpose, many generic assays were developed to amplify genomic fragments within the VP4-, VP2-, or VP1-encoding regions by RT-PCR and to sequence the resulting amplicons. The sequences of clinical or field viruses can be compared to those of prototype strains in order to determine their respective types.

Besides typing, molecular characterization of circulating EVCs generally implies sequencing of untranslated or non-structural genomic regions, or even full-length sequencing of the genome. Indeed, recombination events between enteroviruses are known to be very frequent, thus leading to complex ecosystems of cocirculating viruses featuring mosaic genomes (Savolainen-Kopra and Blomqvist, 2010; Combelas et al., 2011; Kyriakopoulou et al., 2015). As recombination constitutes a powerful force that drives enterovirus evolution, sequencing of different genomic regions of isolates is crucial to detect recombination events. Such events are revealed by incongruent clustering of genetic sequences in phylogenetic trees, depending on the studied genomic regions.

Generic assays have been developed to amplify non-structural regions of EV genomes, particularly the $5^{\prime}$-UTR and the $2 \mathrm{C}$ and the 3D-encoding regions (Bessaud et al., 2008). These widerange assays can be used to characterize portions of the genome of EV-Cs but determining the full-length genomic sequences requires additional sequencing steps to bridge the gaps between the sequences determined through generic assays. These steps require the usage of specific primers that have to be designed for each isolate and can be very labor-intensive when numerous isolates of many types are studied.

In order to facilitate the full-length sequencing of $\mathrm{EV}-\mathrm{C}$ isolates, a simple method was developed, which allowed the amplification of the whole genome by RT-PCR using four generic primer pairs. Four overlapping fragments were produced by performing four separate PCR reactions on cDNAs generated through a single RT reaction. The four amplicons were then pooled and purified prior to sequencing by high-throughput sequencing methods.

The amplification step was assessed on a panel of prototype and field strains whose genome had been previously sequenced by the Sanger method. Sequencing data was analyzed by mapping the reads against reference sequences and by de novo assembly. This method was able to determine the full-length genomic sequences of EV-C isolates with high sensitivity and to discriminate the different genomic sequences within mixtures of viruses.
TABLE 1 | Viruses used in the study.

\begin{tabular}{|c|c|c|c|}
\hline Type & $\begin{array}{l}\text { Strain or } \\
\text { isolate }\end{array}$ & Origin & $\begin{array}{c}\text { Cycle } \\
\text { threshold }^{\mathrm{a}}\end{array}$ \\
\hline CV-A1 & Tompkins & Prototype strain & $14.5 \pm 0.2$ \\
\hline CV-A11 & Belgium & Prototype strain & $16.6 \pm 0.2$ \\
\hline CV-A11 & G9 & Prototype strain & $14.9 \pm 0.7$ \\
\hline CV-A11 & 66122 & From Rakoto-Andrianarivelo et al. (2007) & $15.6 \pm 0.1$ \\
\hline CV-A11 & 66990 & From Rakoto-Andrianarivelo et al. (2007) & $15.9 \pm 0.1$ \\
\hline CV-A13 & Flores & Prototype strain & $15.7 \pm 0.3$ \\
\hline CV-A13 & G13 & Prototype strain & $23.7 \pm 0.1$ \\
\hline CV-A13 & 67900 & From Rakoto-Andrianarivelo et al. (2007) & $17.1 \pm 0.4$ \\
\hline CV-A13 & 67001 & From Rakoto-Andrianarivelo et al. (2007) & $13.3 \pm 0.2$ \\
\hline CV-A17 & G12 & Prototype strain & $13.8 \pm 0.3$ \\
\hline CV-A17 & 67591 & From Rakoto-Andrianarivelo et al. (2007) & $14.4 \pm 0.9$ \\
\hline CV-A17 & 68154 & From Rakoto-Andrianarivelo et al. (2007) & $15.2 \pm 0.2$ \\
\hline CV-A19 & $\mathrm{NIH}-8663$ & Prototype strain & $23.2 \pm 1.0$ \\
\hline CV-A20 & Cecil & Prototype strain & $16.0 \pm 0.1$ \\
\hline CV-A20 & IH35 & Prototype strain & $13.5 \pm 0.4$ \\
\hline CV-A20 & Tulane & Prototype strain & $16.1 \pm 0.1$ \\
\hline CV-A21 & Coe & Prototype strain & $17.7 \pm 0.3$ \\
\hline EV-C95 & T08-083 & From Sadeuh-Mba et al. (2013) & $14.5 \pm 0.5$ \\
\hline EV-C99 & 68229 & From Rakoto-Andrianarivelo et al. (2007) & $11.5 \pm 0.1$ \\
\hline$P V-1$ & Sabin & Vaccine strain & $15.6 \pm 0.1$ \\
\hline PV-2 & Sabin & Vaccine strain & $17.4 \pm 0.3$ \\
\hline PV-3 & Sabin & Vaccine strain & $20.9 \pm 0.1$ \\
\hline
\end{tabular}

${ }^{a}$ Cycle threshold values obtained by a pan-enterovirus real-time RT-PCR assay performed on the extracted RNAs.

\section{MATERIALS AND METHODS}

\section{Viruses}

Twenty-two viruses were used for this study (Table 1). The prototype viruses are available on the Pasteur Institute collection (Centre de ressources biologiques de l'Institut Pasteur, Paris, France). Field isolates originated from stool samples collected in Madagascar in 2002 (Rakoto-Andrianarivelo et al., 2005, 2007) and in Chad in 2008-2009 (Sadeuh-Mba et al., 2013). The whole genomic sequence of these isolates were previously determined by the Sanger method (Bessaud et al., 2011).

All work with infectious viruses was carried out in a BSL2 facility. All viruses were grown in HEp-2c cell monolayers in DMEM supplemented with $2 \%$ fetal calf serum and $2 \mathrm{mM} \mathrm{L-}$ glutamine at $37^{\circ} \mathrm{C}$, except coxsackievirus (CV) A1 Tompkins and CV-A19 NIH-8663, see below. These latter cannot be propagated in cell lines but can infect suckling mice. RNA of these two viruses was extracted from brains of mice inoculated intracerebrally. These brains were retrieved from the laboratory collection and no mice were used during this study. Brains were crushed in PBS before RNA extraction.

\section{Sequence Analysis and Primer Design}

EV-C full-length nucleotide sequences retrieved from the GenBank database were aligned using CLC Main Workbench 7.6.4 software (CLC bio). Eight degenerated primers were designed to target conserved genomic regions (Table 2). 
TABLE 2 | Primers used in this study.

\begin{tabular}{lllc}
\hline Step & Name & $\mathbf{5}^{\prime} \rightarrow \mathbf{3}^{\prime}$ sequence & $\begin{array}{c}\text { Genome } \\
\text { position }\end{array}$ \\
\hline $\begin{array}{l}\text { cDNA } \\
\text { synthesis }\end{array}$ & heptaN & NNNNNNN & \\
PCR & C004 & TAAAACAGCYYKDGGGTTG & $1-20$ \\
& C005 & CCGAATYAAARAAATTTACCC & $7437-7415$ \\
& C008 & CARTTYAGASCAARCAYCG & $4459-4478$ \\
& C009 & ACCATYTGRCARAANARYTTCA & $4703-4682$ \\
& C018 & ATGYTNGGNACNCAYNTNATHTGGGA & $2212-2237$ \\
& C019 & CCYTGYTCCATNRCYTCHTCYTC & $3833-3811$ \\
& C021 & TCDGGNARYTTCCACCACCA & $1205-1186$ \\
& C022 & GARGCNTGYGGDTAYAGYGA & $967-986$
\end{tabular}

${ }^{a}$ Relative to PV-2 strain Sabin.

\section{RNA Extraction}

Viral RNA was extracted from $250 \mu \mathrm{L}$ of culture supernatants or clarified brain extracts using the High Pure Viral RNA kit (Roche Diagnostics, Meylan, France), following the manufacturer's instructions.

In order to check the RNA extraction step, a one-step realtime RT-PCR assay was performed on extraction products using a pan-enterovirus generic assay previously described (Monpoeho et al., 2000).

\section{Synthesis of the Four Overlapping Amplicons by 2-Step RT-PCR}

For each virus, four overlapping DNA fragments were produced by RT-PCR (Figure 1). cDNA synthesis was performed as previously described (Bessaud et al., 2008). The reaction mixture contained $5 \mu \mathrm{L}$ of purified viral RNA, $2 \mu \mathrm{L}$ of $5 \mathrm{X}$ First-Strand Buffer, $0.01 \mathrm{M}$ dithiothreitol $(1 \mu \mathrm{L}), 100 \mathrm{ng}$ of the random primers heptaN $(1 \mu \mathrm{L}), 10 \mathrm{nmol}$ of each dNTP $(1 \mu \mathrm{L}$ of a $10 \mathrm{mM}$ mixture), and $100 \mathrm{U}$ of SuperScript II $(0.5 \mu \mathrm{L})$. The RT reaction mixture was incubated at $25^{\circ} \mathrm{C}$ for $10 \mathrm{~min}, 42^{\circ} \mathrm{C}$ for $45 \mathrm{~min}$ and $95^{\circ} \mathrm{C}$ for $5 \mathrm{~min}$.

The cDNA was then used as a template for amplification in four PCRs carried out in a final volume of $50 \mu \mathrm{L}$ that included $5 \mu \mathrm{L}$ of 10X PCR Buffer w/o $\mathrm{MgCl}_{2}, 1.5 \mathrm{mM}$ of $\mathrm{MgCl}_{2}, 10 \mathrm{nmol}$ of each dNTP, 50 pmol of each primer, $2 \mu \mathrm{L}$ of cDNA, and $2.5 \mathrm{U}$ of Platinum Taq DNA polymerase (Invitrogen). The thermocycler profile was $2 \mathrm{~min}$ at $94^{\circ} \mathrm{C}$ followed by 30 cycles of $30 \mathrm{~s}$ at $94^{\circ} \mathrm{C}$, $30 \mathrm{~s}$ at $55^{\circ} \mathrm{C}$, and $3 \mathrm{~min}$ at $72^{\circ} \mathrm{C}$.

Ten microliters of each PCR product were analyzed on ethidium bromide-stained agarose gels. For each virus, the four PCR products were pooled, purified on silica columns (Wizard SV Gel and PCR Clean-Up System, Promega) and eluted in $30 \mu \mathrm{L}$ of water. No purification of the amplicons was performed by gelexcision, even when additional bands were detected on ethidium bromide-stained agarose gels.

\section{Assay Sensitivity}

The sensitivity of the assay was evaluated using serial threefold dilutions of a cell culture supernatant infected by CVA13 isolate 67001 whose titer was determined according to the WHO standard protocol (Anonymous, 2004). To maintain the same amount of cellular nucleic acids across dilutions, dilutions were prepared using a supernatant of confluent noninfected HEp-2c cell monolayer that was frozen and thawed twice and then clarified by centrifugation. RNA was extracted from $250 \mu \mathrm{L}$ of each dilution and subjected to real-time RT-PCR as described in Section RNA Extraction, and amplified by RT and PCR as described in Section Synthesis of the Four Overlapping Amplicons by 2-Step RT-PCR.

\section{Detection of Virus Mixtures}

Four mixtures were prepared using cell culture supernatants of CV-A13 isolates 67900 and 67001 whose titer was determined according to the WHO standard protocol (Anonymous, 2004). Titers were adjusted to $10^{7.5} \mathrm{TCID}_{50} \cdot \mathrm{mL}^{-1}$ before mixing. Different mixtures were prepared with 67900/67001 titer ratios ranging from 1:1 to 10:1. RNA was extracted from $250 \mu \mathrm{L}$ of these mixtures and subjected to RT and PCR as described in Section Synthesis of the Four Overlapping Amplicons by 2-Step RT-PCR.

\section{Sequencing Process}

DNA concentration of the purified RT-PCR products was determined by using a VarioskanLux (ThermoScientific). Libraries were built using $1 \mathrm{ng}$ of DNA with the Nextera XT DNA Library Preparation kit in a SureCycler 8800 thermocycler (Agilent). After purification on AMPure beads (Beckman), the libraries were controlled using the High Sensitivity D1000 assay (Agilent) on a TapeStation 2200. Sizing was achieved by electrophoresis on a PippinPrep System with the PippinPrep kit CDF1510 (Ozyme). Finally, the libraries were quantified using the KAPA Quantification kit on a LightCycler 96 System (Roche). Sequencing was performed on a NextSeq500 with the HighOutput or MidOutput kits. All kits were used following manufacturer's instructions.

\section{Data Analysis}

\section{Trimming}

Reads were demultiplexed followed by the removal of tags and adaptors. After importation in CLC Genomics Workbench 8.5 (CLCbio), reads were trimmed using the following parameters: Trim quality score limit $=0.01$; Trim ambiguous nucleotides with Maximum number of ambiguities $=1$. To avoid the presence of primer sequences in the reads, the $265^{\prime}$ - and $3^{\prime}$-terminal nucleotides were removed. After trimming, reads shorter than 50 nucleotides were discarded.

\section{Mapping of the Reads to a Reference}

For each sample, the trimmed reads were mapped against the reference sequence of the corresponding virus. The sequences used as references were the sequences previously determined by the Sanger method. The entire genome of all field isolates were previously sequenced in our laboratory (Bessaud et al., 2011); for prototype strains, the sequences used as references were retrieved from the Genbank database. Mapping was achieved with CLC Genomics Workbench 8.5 by using the following parameters: Mismatch cost $=10$; Insertion cost $=3$; Deletion cost $=3$; Length fraction $=0.5$; Similarity fraction $=0.95$. 


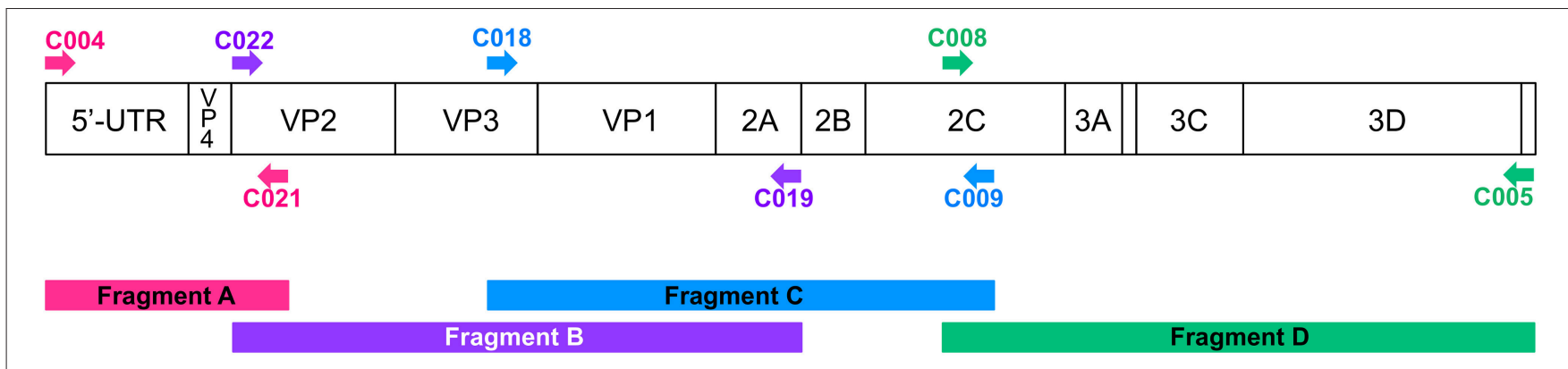

FIGURE 1 | Schematic representation of the locations of the primers used for the PCR step. The upper scheme represents the organization of the enterovirus genomic RNA. Arrows indicate the sites targeted by the primers. The RT-PCR products are colored according to the corresponding primer pair.

\section{De novo Assembly}

For each sample, the trimmed reads were assembled without any reference using CLC Genomics Workbench 8.5 with the following parameters: Mismatch cost $=2$; Insertion cost $=2$; Deletion cost $=2$; Length fraction $=0.5$; Similarity fraction $=$ 0.95 .

All contigs longer than 200 nucleotides were submitted to BLAST analysis (Mount, 2007). Virus contigs were compared with the reference sequence of the corresponding virus determined by the Sanger method through alignment.

\section{RESULTS}

\section{Sequence Analysis and Primer Design}

Eight degenerated primers were designed to target nucleotide sequences that were conserved among EV-Cs (Figure 1). C004 and C005 target the extremity of the $5^{\prime}$ and $3^{\prime}$ non-coding region, respectively. C018, C019, and C008 match with conserved regions already targeted by pan-EV molecular assays (Caro et al., 2001; Nix et al., 2006; Bessaud et al., 2008) within the VP3 gene, at the $2 \mathrm{~A} / 2 \mathrm{~B}$ junction, and within the $2 \mathrm{C}$ cis-acting replication element (Cordey et al., 2008), respectively. The three other primers, C021, C022, and C009 match with VP2 and 2C sequences.

The primer pairs C004/C021, C022/C019, C018/C009, and C008/C005 were used to produce four overlapping DNA fragments (fragments $\mathrm{A}-\mathrm{D}$ ) that span the entire viral genome. Fragments ranged from $\sim 1200$ to $\sim 3000$ nucleotides in length. Overlapping regions were approximately 240-nt-long for fragments $\mathrm{A}$ and $\mathrm{B}$ and fragments $\mathrm{C}$ and $\mathrm{D}$ (Figure 1). The overlapping region for fragments $\mathrm{B}$ and $\mathrm{C}$ was longer than 1600 nt.

\section{RT-PCR Amplification}

The four primer pairs C004/C021, C022/C019, C018/C009, and $\mathrm{C} 008 / \mathrm{C} 005$ were tested on prototype and field strains representative of $12 \mathrm{EV}-\mathrm{C}$ types (Table 1). This panel included the three PV serotypes and non-polio types commonly isolated during environmental or epidemiological surveillance, such as CV-A11, CV-A13, CV-A17, CV-A20, CV-A21, and EV-C99. One field strain belonged to the type EV-C95 that was recently discovered (Junttila et al., 2015) and of which few isolates were reported in Africa. The panel also included the prototype strains of CV-A1 and CV-A19, two types that are uncultivable on cell lines (Brown et al., 2003). Overall, 22 viruses were used to assess the efficiency of the primers. After RNA extraction, detection of viral RNA by real-time RT-PCR resulted in cycle threshold values ranging from $11.5 \pm 0.1$ to $23.7 \pm 0.1$ (Table 1 ).

Following the RT step with random primers performed on viral RNA extracted from infected cell culture supernatants (or from crushed brains of mice infected by CV-A1 or CV-A19), the PCR performed with the four primer pairs produced gel bands at the expected size for all the viruses (data not shown). Some additional bands were also observed for certain RT-PCR products. Among our panel, CV-A19 NIH-8663 RNA gave the weakest bands on ethidium bromide-stained gel after the RTPCR step. It is not possible to exclude the hypothesis that the four primer pairs had a low efficiency in amplifying the genome of this virus through PCR because of mismatches. Nonetheless, since the RNA of this virus was extracted from a mouse brain collected more than 20 years ago, this result is more likely due to the low amount of full-length virus RNA in this sample.

\section{Results of Sequencing}

The products of RT-PCR amplification of non-polio EV were sequenced by Illumina technology. Due to French regulations relating to PV containment, the sequencing platform was not allowed to handle the PV RT-PCR products.

For each sample, the four RT-PCR fragments were produced separately and then pooled prior to purification. Sequencing resulted in a list of reads of which most were $\sim 100$ nucleotidelong after trimming. Because all of the samples were not sequenced during the same run, the number of reads varied from sample to sample. Thus, the number of reads after trimming ranged from 76,802 to 714,322 (Table 3).

\section{Bioinformatics Analysis}

Raw data generated by high-throughput sequencing was analyzed by two different methods commonly used for virus characterization.

The first method consists in mapping the reads against a reference sequence. This method can be used to re-sequence isolates or to detect single-point mutations that appear when a given virus is propagated under different conditions (e.g., various 
TABLE 3 | Overview of the results of the bioinformatics analysis performed on sequencing data.

\begin{tabular}{|c|c|c|c|c|c|}
\hline \multirow[t]{2}{*}{ Strain or isolate } & \multirow[t]{2}{*}{ Total number of reads } & \multicolumn{2}{|c|}{ Mapping on reference sequence } & \multicolumn{2}{|c|}{ De novo assembly } \\
\hline & & $\begin{array}{c}\text { Number of mapped } \\
\text { reads }\left(\%^{a}\right)\end{array}$ & $\begin{array}{l}\text { Average } \\
\text { depth }\end{array}$ & $\begin{array}{l}\text { Number of reads in } \\
\text { the virus contig }\left(\%^{b}\right)\end{array}$ & $\begin{array}{c}\text { Number of } \\
\text { host contigs }\end{array}$ \\
\hline \multicolumn{6}{|l|}{ CV-A1 } \\
\hline Tompkins & 391,846 & 380,207 (97\%) & 7032 & 379,991 (96\%) & 200 \\
\hline \multicolumn{6}{|l|}{ CV-A11 } \\
\hline Belgium & 373,748 & 337,637 (90\%) & 6214 & 337,708 (90\%) & 232 \\
\hline G9 & 121,080 & 119,260 (98\%) & 2310 & 120,181 (99\%) & 16 \\
\hline 66122 & 76,802 & $76,342(99 \%)$ & 982 & $76,290(99 \%)$ & 3 \\
\hline 66990 & 121,784 & $114,961(94 \%)$ & 2277 & $120,712(99 \%)$ & 10 \\
\hline \multicolumn{6}{|l|}{ CV-A13 } \\
\hline Flores & 88,094 & $86,883(97 \%)$ & 1608 & $81,912(93 \%)$ & 6 \\
\hline G13 & 101,372 & $98,056(96 \%)$ & 1902 & $100,746(99 \%)$ & 12 \\
\hline 67900 & 175,426 & 174,488 (99\%) & 3382 & 154,526 (88\%) & 1 \\
\hline 67001 & 189,890 & 185,674 (97\%) & 3689 & 188,303 (99\%) & 0 \\
\hline \multicolumn{6}{|l|}{ CV-A17 } \\
\hline G12 & 554,763 & 553,436 (99\%) & 10,404 & 553,221 (99\%) & 28 \\
\hline 67591 & 421,668 & 367,691 (87\%) & 4741 & 365,019 (87\%) & 197 \\
\hline 68154 & 84,786 & 83,710 (98\%) & 1548 & $83,251(98 \%)$ & 14 \\
\hline \multicolumn{6}{|l|}{ CV-A19 } \\
\hline $\mathrm{NIH}-8663$ & 374,976 & 153,262 (40\%) & 2958 & 152,980 (40\%) & 783 \\
\hline \multicolumn{6}{|l|}{ CV-A20 } \\
\hline Cecil & 619,194 & 616,252 (99\%) & 11,525 & 615,858 (99\%) & 59 \\
\hline IH35 & 698,498 & 630,711 (99\%) & 11,657 & 632,064 (99\%) & 39 \\
\hline Tulane & 714,322 & 712,310 (99\%) & 13,336 & 712,445 (99\%) & 21 \\
\hline \multicolumn{6}{|l|}{ CV-A21 } \\
\hline Coe & 570,328 & 525,606 (92\%) & 9608 & 565,659 (99\%) & 115 \\
\hline \multicolumn{6}{|l|}{ EV-C95 } \\
\hline T08-083 & 96,460 & 96,059 (99\%) & 1776 & 96,110 (99\%) & 7 \\
\hline \multicolumn{6}{|l|}{ EV-C99 } \\
\hline 68229 & 575,410 & 573,084 (99\%) & 10,435 & 572,958 (99\%) & 19 \\
\hline
\end{tabular}

a These percentages indicate the proportion of total reads that matched with the reference sequence.

$b$ These percentages indicate the proportion of total reads that were included in the virus contig through de novo assembly.

host species, various cell lines or presence of antiviral molecules, for example).

The second method consists in constructing contigs through de novo assembly (i.e., without any reference sequence to drive the assembly). This method can be used to determine the full-length sequence of viruses isolated from clinical or environmental samples.

\section{Mapping on Reference Sequence}

For each sample, reads were mapped against the corresponding sequence previously determined by the Sanger method (Table 3). For all samples except one, more than $87 \%$ of the reads mapped against the reference sequence. The only exception was CV-A19 NIH-8663, a non-cultivable virus, in which only $40 \%$ of the reads mapped to the reference.

For all samples, the reference sequence was fully covered without any gaps (breadth of coverage $>99.0 \%$ ). The breadth of coverage never reached $100.0 \%$ because the sequences corresponding to the outer primers $\mathrm{C} 004$ and $\mathrm{C} 005$ and the few nucleotides located downstream C005 were absent in the final contig.

Average depth of coverage, which is the average number of times a base is sequenced, was very high $(>980)$ but varied greatly from sample to sample (Table 3), depending on the total number of reads (correlation coefficient $>0.999$ ). Coverage depth was heterogeneous along the mapping (Figure 2) but was high enough to deduce an unambiguous consensus sequence even for samples with a low number of reads (CV-A11 66122 or CVA17 68154 for instance) or a low proportion of mapping reads (CV-A19 NIH-8663).

The consensus sequences of field strains deduced from mapping were compared to the consensus sequences determined by the Sanger method using RNA extracted from the same cell culture supernatants. For each virus, the two consensus sequences were identical indicating that high-throughput sequencing did not introduced nucleotide 


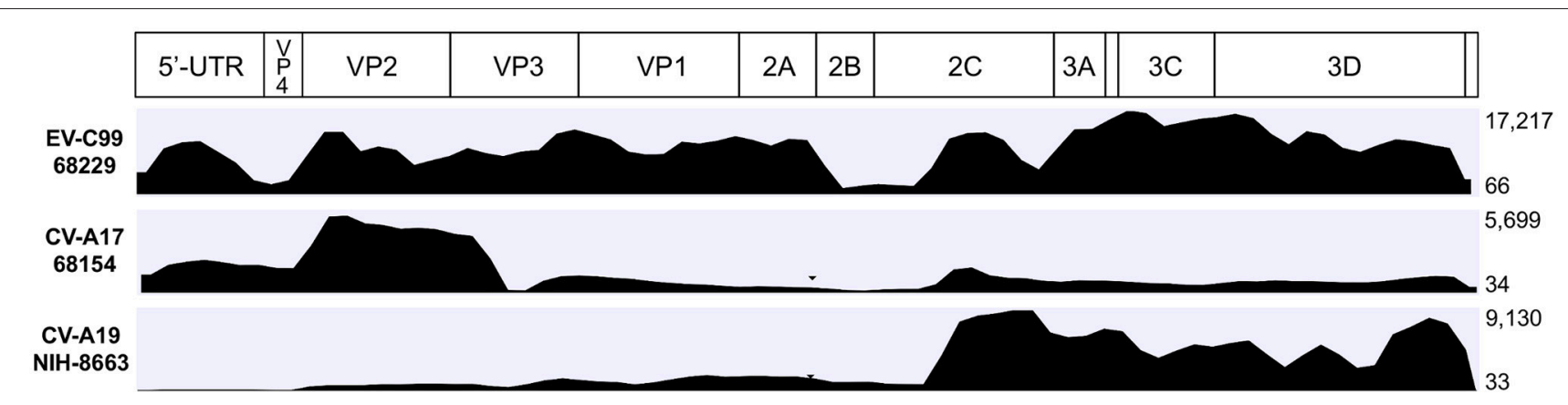

FIGURE 2 | Coverage depth observed for three samples after mapping on their respective reference sequence. Coverage depth is the number of times a base is sequenced. On the right side of each graph, the minimum and maximum coverage depth values are indicated.

changes in the consensus sequences compared to the Sanger method.

\section{De novo Assembly}

In order to assess the ability of the method to determine fulllength sequences of the original isolates, the trimmed reads of each sample were assembled without using a reference.

For all samples, the de novo assembly tool of CLC Genomics Workbench succeeded in assembling the virus genome into a unique contig that covered the entire genome between the outer primers C004 and C005. The number of reads included in the final full-length contigs was virtually similar to the number of reads previously found to map against the reference (Table 3 ). The consensus sequence deduced from de novo assembly was identical to the consensus sequence already determined by mapping.

For most samples, besides the viral contigs, de novo assembly produced additional contigs that were identified as human sequences (or mouse sequences for CV-A1 and CV-A19 samples) by BLAST analysis. These contigs were from non-specific amplification of cellular nucleic acids during the RT-PCR process or from contamination of the viral RNA with cellular DNA during extraction. The number of such contigs varied tremendously from sample to sample, ranging from 0 to 783 (Table 3). Nonetheless, contaminating nucleic acids did not compromise the proper assembly of the viral genome, even for the CV-A19 sample that contained mostly non-viral reads.

\section{Sensitivity of the Assay}

In order to evaluate the sensitivity of the assay, three-fold serial dilutions of a CV-A13 67001 supernatant at $10^{7.8} \mathrm{TCID}_{50} \cdot \mathrm{mL}^{-1}$ were prepared. After RNA extraction, detection of viral RNA by real-time RT-PCR showed positive results for extracts from the first 15 dilutions (Table 4).

The 2-step RT-PCR assays using C004/C021, C022/C019, C018/C009, and C008/C005 were tested on these extracts. The four primer pairs were able to generate detectable amplicons for RNA extracted from the undiluted supernatant and from the first four dilutions (Figure 3). Since ethidium bromide-stained gels have a detection limit of few nanograms of DNA per band, the absence of visible bands after the amplification of the highest dilutions does not indicate necessarily that no amplicons were generated for these dilutions. Therefore, all the RT-PCR products were investigated through high-throughput sequencing.

After pooling and purification, the RT-PCR products of each dilution were sequenced during the same run. The total number of reads varied from sample to sample, from 88,930 to 316,336 (Table 4). In the sample from the undiluted supernatant, $99.9 \%$ of reads mapped against the reference sequence. As expected this proportion decreased when the dilution factor increased but even the samples from the highest dilutions contained a few hundred reads from the virus genome. The non-mapped reads were from cellular nucleic acids. For the six last samples (dilution factor $\geq 3^{10}$ ), the number of virus reads was too low to reconstitute the full-length genome sequence through de novo assembly. By contrast, de novo assembly succeeded in building the full-length genome for all samples with dilution factor $\leq 3^{9}$, including the last one in which only $2.5 \%$ of the reads were from the virus. For this sample, the coverage depth ranged from 5 to 95 along the contig (average of 28). In spite of this relatively low depth, the contig sequence generated for this sample was identical to the Sanger consensus sequence.

These results demonstrated that the method was able to generate full-length sequences of EV-C isolates from a few thousand reads and indicated that sequencing can be attempted even in the absence of detectable bands on agarose gels.

\section{Detection of Mixtures}

Supernatants of cell cultures inoculated with clinical and environmental samples often contain mixtures of enteroviruses. In order to determine whether the method was able to sequence mixtures by assembling properly different viral contigs, four mixtures of viruses were prepared by mixing cell culture supernatants of two viruses, 67900 and 67001 . These two viruses belong to the same serotype (CV-A13) and display together a nucleotide identity of $83 \%$. The four mixtures contained the same volume of 67900 supernatant but different volumes of 67001 supernatant (Table 5). Thus, the titer ratios ranged from 1:1 to $10: 1$.

For the four mixtures, de novo assembly generated separate contigs for reads from 67900 and from 67001. As expected, the proportion of reads included in the 67900 contigs increased from mixture 1 to mixture 4 while the proportion of reads in the 67001 contigs decreased (Table 5). Nonetheless, even in mixture 
TABLE 4 | Evaluation of the sensitivity of the assay.

\begin{tabular}{|c|c|c|c|c|c|c|}
\hline $\begin{array}{l}\text { Dilution } \\
\text { factor }\end{array}$ & $\begin{array}{c}\text { Viral titer } \\
\left(\mathrm{TCID}_{50} \cdot \mathrm{mL}^{-1}\right)\end{array}$ & $\begin{array}{l}\text { RT-PCR cycle } \\
\text { threshold }\end{array}$ & $\begin{array}{l}\text { DNA concentration } \\
\qquad\left(\mathrm{ng} \cdot \mu \mathrm{L}^{-1}\right)^{\mathrm{b}}\end{array}$ & $\begin{array}{l}\text { Number of } \\
\text { reads }\end{array}$ & $\begin{array}{l}\text { Number of reads mapping } \\
\text { against the reference }\left(\%{ }^{c}\right)\end{array}$ & $\begin{array}{l}\text { Construction of a full-length } \\
\text { contig through de novo assembly }\end{array}$ \\
\hline $3^{0}$ & $10^{7.8}$ & $16.2 \pm 0.1$ & $>200.0$ & 197,190 & 196,993 (99.9\%) & Yes \\
\hline $3^{1}$ & $10^{7.3}$ & $17.9 \pm 0.4$ & 90.4 & 143,770 & 143,125 (99.5\%) & Yes \\
\hline $3^{2}$ & $10^{6.8}$ & $20.1 \pm 0.4$ & 76.7 & 140,554 & 138,103 (98.2\%) & Yes \\
\hline $3^{3}$ & $10^{6.4}$ & $21.6 \pm 0.2$ & 18.7 & 112,070 & 104,730 (93.4\%) & Yes \\
\hline $3^{4}$ & $10^{5.9}$ & $23.3 \pm 0.1$ & 6.8 & 190,346 & 172,273 (90.5\%) & Yes \\
\hline $3^{5}$ & $10^{5.4}$ & $25.1 \pm 0.1$ & 5.5 & 316,336 & $218,511(69.1 \%)$ & Yes \\
\hline $3^{6}$ & $10^{4.9}$ & $26.7 \pm 0.1$ & 3.9 & 216,594 & 117,302 (54.1\%) & Yes \\
\hline $3^{7}$ & $10^{4.5}$ & $28.4 \pm 0.2$ & 3.4 & 117,920 & 48,054 (27.0\%) & Yes \\
\hline $3^{8}$ & $10^{4.0}$ & $30.0 \pm 0.2$ & 3.8 & 130,334 & 14,828 (11.3\%) & Yes \\
\hline $3^{9}$ & $10^{3.5}$ & $31.6 \pm 0.1$ & 2.8 & 88,930 & $2282(2.5 \%)$ & Yes \\
\hline $3^{10}$ & $10^{3.0}$ & $33.4 \pm 0.2$ & 2.5 & 119,950 & 1109 (0.9\%) & No \\
\hline $3^{11}$ & $10^{2.6}$ & $35.2 \pm 0.6$ & 3.1 & 104,470 & $511(0.4 \%)$ & No \\
\hline $3^{12}$ & $10^{2.1}$ & $36.1 \pm 0.3$ & 2.7 & 124,876 & $297(0.2 \%)$ & No \\
\hline $3^{13}$ & $10^{1.6}$ & $37.5 \pm 0.6$ & 1.1 & 105,656 & $208(0.2 \%)$ & No \\
\hline $3^{14}$ & $10^{1.1}$ & $>38.0^{\mathrm{a}}$ & 1.5 & 176,260 & $314(0.2 \%)$ & No \\
\hline $3^{15}$ & $10^{0.6}$ & $>39.0^{a}$ & 1.1 & 111,890 & 228 (0.2\%) & No \\
\hline
\end{tabular}

a Only one well of the triplicate assay showed a detectable increase in fluorescence.

${ }^{b}$ After pooling of the four RT-PCR products and purification, the DNA concentration was measured on a VarioskanLux spectrophotometer.

${ }^{c}$ These percentages indicate the proportion of total reads that matched with the reference sequence.

4, which contained 10-fold less starting 67001 RNA compared to mixture 1 , the method was able to properly generate a full-length contig corresponding to the expected sequence of 67001.

These results demonstrated that, by using the parameters indicated in Section De novo Assembly, the stringency of the de novo bioinformatics analysis was high enough to allow the segregation of the reads into separate contigs.

\section{DISCUSSION}

High-throughput sequencing techniques constitute a powerful tool for the study of viruses (Quiñones-Mateu et al., 2014; Nelson and Hughes, 2015). By allowing concomitant sequencing of millions of DNA fragments, they allow rapid sequencing of a great number of samples and in-depth characterization of minority genomic variants. The aim of this study was to develop a convenient method allowing the whole sequencing of the genome of EV-C isolates by using high-throughput sequencing.

Different strategies have been reported to copy viral genomic RNAs into DNA fragments that can be subsequently sequenced by high-throughput techniques.

Some strategies are based on random amplification using non-specific primers (Berthet et al., 2008; Djikeng et al., 2008). These strategies generally lead to the generation of DNA libraries that mainly consist of non-viral sequences, thus decreasing the amount of relevant reads obtained by sequencing. Reducing the amount of unwanted reads requires the use of additional procedures to physically enrich the samples for viral RNAs (Hall et al., 2014) or to limit the amplification of host nucleic acids (Ge et al., 2015). Whole sequencing of virus RNA genomes amplified by random primers can also be impaired by the relatively low amplification rate of some genomic regions, which can lead to gaps in the genomic sequences (Rosseel et al., 2013).

Alternate strategies are based on primers that specifically target the viral RNAs to be sequenced. Such strategies have been already reported to sequence several positive-strand RNA viruses, including enterovirus A71 (Wright et al., 2011; Baronti et al., 2015; Cruz et al., 2016; Thomson et al., 2016). In our experiments, using EV-C-targeting primers rather than random primers limited the number of reads from cellular nucleic acids: the proportion of reads from viral origin was higher than $90 \%$ for most samples. The method was thus sensitive enough to determine full-length genomes through de novo assembly from only a few thousand reads.

The four primer pairs were efficiently tested on viruses belonging to EV-C types commonly isolated during epidemiological studies, including the three serotypes of PV. They also amplified CV-A1 and CV-A19, which were more closely related to EV-C types recently identified, such as EV-C113 and EV-C116 (Tokarz et al., 2013). Since the primers were designed from an alignment comprising the sequences of all currently known EV-C types, they are likely to be used successfully to amplify any EV-Cs. We cannot exclude that these primers are also able to amplify genomic sequences of viruses closely related to EV-Cs. In particular, the primer C008 targets the cis-acting replication element that is highly conserved among EV-As, -Bs, -Cs, and -Ds (Cordey et al., 2008). Reads from EVAs, -Bs, or -Ds, or even rhinoviruses could thus be found in the sequencing data generated from EV-Cs cell cultute supernatants co-infected by members of these species. Nonetheless, as our bioinformatics analyses was stringent enough to discriminate reads from two EV-Cs belonging to the same type, they are likely to discriminate reads from more divergent viruses, thereby 

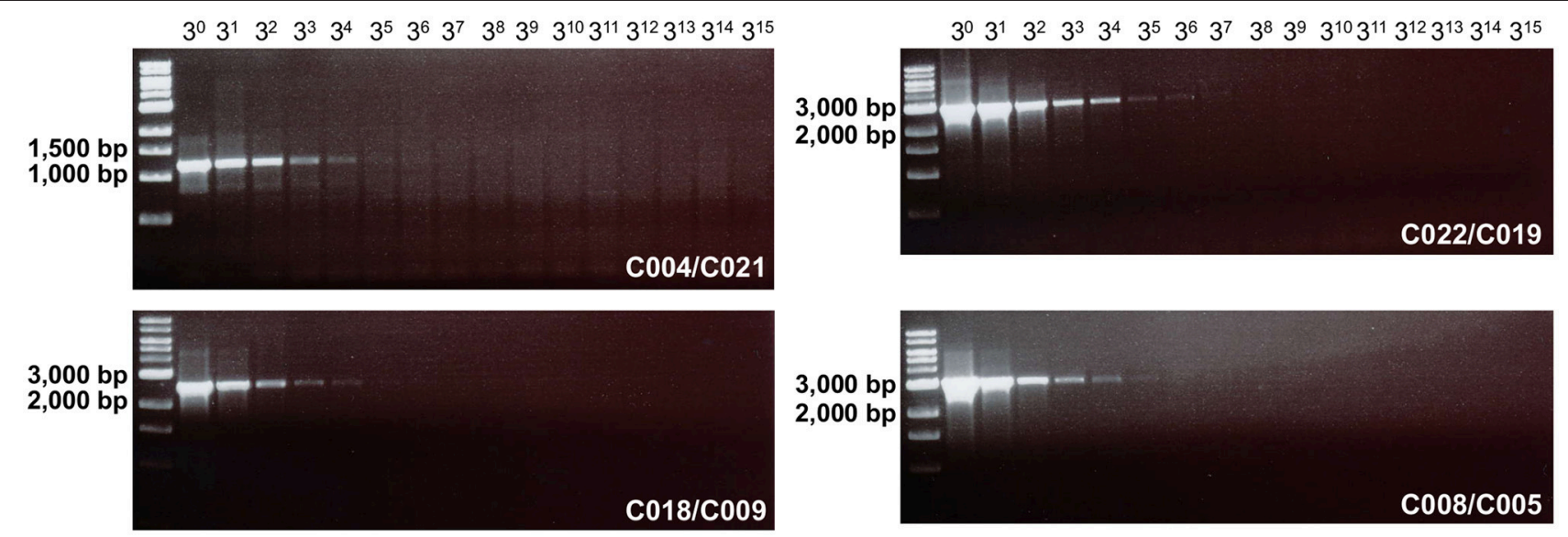

FIGURE 3 | Sensitivity of the different primer pairs on CV-A13 67001 RNA extracted from three-fold serial dilutions. The DNA size scale, expressed in base pairs (bp), is indicated on the left side of the gels.

TABLE 5 | Results obtained after sequencing and de novo assembly of mixtures of two viruses, CV-A13 67900 and CV-A13 67001.

\begin{tabular}{|c|c|c|c|c|c|c|c|}
\hline \multicolumn{3}{|c|}{ Viral titer $\left(\log _{10} \mathrm{TCID}_{50}\right)$} & \multirow[t]{2}{*}{ Total number of reads } & \multicolumn{4}{|c|}{ De novo assembly } \\
\hline & & & & Reads $\left(\%{ }^{a}\right)$ & Average depth & Reads $\left(\%^{b}\right)$ & Average depth \\
\hline 7.5 & 7.5 & $1: 1$ & 267,226 & 84,674 (31\%) & 1540 & $178,860(66 \%)$ & 3396 \\
\hline 7.5 & 7.2 & $2: 1$ & 224,848 & 94,097 (41\%) & 1689 & 121,239 (53\%) & 2313 \\
\hline
\end{tabular}

${ }^{a}$ These percentages indicate the proportion of total reads that were included in the CV-A13 67900 contig through de novo assembly.

${ }^{b}$ These percentages indicate the proportion of total reads that were included in the CV-A13 67001 contig through de novo assembly.

preventing the generation of contigs made of reads from viruses belonging to different species.

Our method was able to generate sequencing data even when no bands were observed on agarose gel after the RT-PCR step. This is a great advantage compared to Sanger-based sequencing that requires substantial amounts of DNA. Since the sensitivity of high-throughput sequencing depends as much on the sequencing depth (i.e., the total number of reads obtained for a given sample) as it does on the amount of virus genome copies in the sample, increasing the sequencing depth would overcome the low yield of RT-PCR amplification that could be observed for some samples. Another advantage of high-throughput sequencing compared to Sanger-based technics is that no gel-purification of the RT-PCR products was required, even in the presence of contaminating bands. In case of numerous samples to be analyzed, the RT-PCR products could be purified faster by using ultrafiltration-based or silica-based 96-well plates rather than individual silica-based columns.

For all samples, the coverage depth was heterogeneous along the genome. Heterogeneous coverage depths are often observed after high-throughput sequencing, partly because of the processing of the samples (Head et al., 2014; van Dijk et al., 2014). Thus, biases can be introduced by the random shearing of the DNA being sequenced (Poptsova et al., 2014) and by the PCR amplification performed during the library preparation (Aird et al., 2011). After these steps, some genomic regions can be overrepresented whereas others are underrepresented in the final libraries. Therefore, the coverage depth along the genome does not reflect necessarily the relative abundance of DNA amplicons in the original sample. However, in our experiments, the low coverage depth observed in some genomic regions did not impair the generation of the full-length consensus sequence of the corresponding genomes through de novo assembly.

In conclusion, we developed a set of generic primers for the synthesis of RT-PCR products that span the whole genome of EV-C isolates. This method was evaluated by using a panel of viruses already characterized by Sanger-based methods to allow the comparison of the sequences generated by our technique with those obtained previously. For de novo assembly, the raw data were analyzed in real conditions, i.e., with no reference to the sequences already obtained by Sanger-based methods. After assembly, the consensus sequences generated in this way were identical to the Sanger consensus sequences.

In this work, the RT-PCR products were sequenced by the Illumina sequencing technology but could be sequenced 
on any high-throughput sequencing platform. Thus, the sequencing of DNA amplicons covering the whole genome of enterovirus A71 isolates on an Ion Torrent Personal Genomic Machine System was previously reported (Baronti et al., 2015).

This method will serve as a multipurpose tool for laboratories involved in the enterovirus surveillance. It could be used to quickly determine full-length genomic sequences of EV-Cs isolated in cell cultures during environmental surveillance or from patients. In particular, characterization of the genome of vaccine-derived $\mathrm{PV}$, which generally display recombinant genomes made of PV and non-PV genetic sequences (Combelas et al., 2011), could be achieved quickly. The method could also be used to get the full-length sequences of EV-Cs belonging to uncharacterized collections, for example those constituted by the laboratories involved in diagnosis or environmental surveillance (Zaidi et al., 2016). Analyzing large panels of full-length EV-C genomes originating from such collections would help to describe the recombination events that occur between co-circulating viruses and to better understand how recombination drives EV-C evolution.

\section{REFERENCES}

Aird, D., Ross, M. G., Chen, W. S., Danielsson, M., Fennell, T., Russ, C., et al. (2011). Analyzing and minimizing PCR amplification bias in Illumina sequencing libraries. Genome Biol. 12:R18. doi: 10.1186/gb-201112-2-r18

Anonymous (2004). "Titration of laboratory quality control standard," in Polio Laboratory Manual, ed WHO (Geneva: World Health Organization), 75-78.

Baronti, C., Piorkowski, G., Leparc-Goffart, I., de Lamballerie, X., and DubotPeres, A. (2015). Rapid next-generation sequencing of dengue, EV-A71 and RSV-A viruses. J. Virol. Methods 226, 7-14. doi: 10.1016/j.jviromet.2015. 09.004

Berthet, N., Reinhardt, A. K., Leclercq, I., van Ooyen, S., Batejat, C., Dickinson, P., et al. (2008). Phi29 polymerase based random amplification of viral RNA as an alternative to random RT-PCR. BMC Mol. Biol. 9:77. doi: 10.1186/1471-21999-77

Bessaud, M., Jegouic, S., Joffret, M. L., Barge, C., Balanant, J., GouandjikaVasilache, I., et al. (2008). Characterization of the genome of human enteroviruses: design of generic primers for amplification and sequencing of different regions of the viral genome. J. Virol. Methods 149, 277-284. doi: 10.1016/j.jviromet.2008.01.027

Bessaud, M., Joffret, M. L., Holmblat, B., Razafindratsimandresy, R., and Delpeyroux, F. (2011). Genetic relationship between cocirculating Human enteroviruses species C. PLOS ONE 6:e24823. doi: 10.1371/journal.pone. 0024823

Blomqvist, S., and Roivainen, M. (2016). Isolation and characterization of enteroviruses from clinical samples. Methods Mol. Biol. 1387, 19-28. doi: 10.1007/978-1-4939-3292-4_3

Brown, B., Oberste, M. S., Maher, K., and Pallansch, M. A. (2003). Complete genomic sequencing shows that polioviruses and members of human enterovirus species $\mathrm{C}$ are closely related in the noncapsid coding region. J. Virol. 77, 8973-8984. doi: 10.1128/JVI.77.16.8973-8984.2003

Caro, V., Guillot, S., Delpeyroux, F., and Crainic, R. (2001). Molecular strategy for 'serotyping' of human enteroviruses. J. Gen. Virol. 82(Pt 1), 79-91. doi: 10.1099/0022-1317-82-1-79

Combelas, N., Holmblat, B., Joffret, M. L., Colbere-Garapin, F., and Delpeyroux, F. (2011). Recombination between poliovirus and coxsackie A viruses of species C: a model of viral genetic plasticity and emergence. Viruses 3, 1460-1484. doi: $10.3390 / \mathrm{v} 3081460$

\section{AUTHOR CONTRIBUTIONS}

Conceived and designed the experiments: $\mathrm{MB}$ and FD. Performed the experiments: MB, SS, MJ, PP. Analyzed the data: MB. Contributed reagents/materials/analysis tools: RR, RV, NJ. Wrote the manuscript: $\mathrm{MB}, \mathrm{FD}$. Critical revision: $\mathrm{MA}, \mathrm{BB}, \mathrm{NJ}$.

\section{FUNDING}

This work was supported by the Institut Pasteur (PTR 484), the Fondation Total, and the US Department of Health and Human Services (grant No. 5 IDSEP140020-02-00). Patsy Polston is granted by a Pasteur Foundation's Gillings Pasteur Fellowship.

\section{ACKNOWLEDGMENTS}

The authors are indebted to Laura Brinas, Andreea Alexandru Maud Vanpeene, Sobhy Wilhame, and Vincent Enouf (Institut Pasteur, Pasteur International Bioresources network (PIBnet), Plateforme de microbiologie mutualisée (P2M), Paris, France) for performing the sequencing experiments.

Cordey, S., Gerlach, D., Junier, T., Zdobnov, E. M., Kaiser, L., and Tapparel, C. (2008). The cis-acting replication elements define human enterovirus and rhinovirus species. RNA 14, 1568-1578. doi: 10.1261/rna.1031408

Cruz, C. D., Torre, A., Troncos, G., Lambrechts, L., and Leguia, M. (2016). Targeted full-genome amplification and sequencing of dengue virus types 1-4 from South America. J. Virol. Methods 235, 158-167. doi: 10.1016/j.jviromet.2016. 06.001

Djikeng, A., Halpin, R., Kuzmickas, R., Depasse, J., Feldblyum, J., Sengamalay, N., et al. (2008). Viral genome sequencing by random priming methods. BMC Genomics 9:5. doi: 10.1186/1471-2164-9-5

Ge, F., Parker, J., Chul Choi, S., Layer, M., Ross, K., Jilly, B., et al. (2015). Preferential amplification of pathogenic sequences. Sci. Rep. 5:11047. doi: 10.1038/srep 11047

Hall, R. J., Wang, J., Todd, A. K., Bissielo, A. B., Yen, S., Strydom, H., et al. (2014). Evaluation of rapid and simple techniques for the enrichment of viruses prior to metagenomic virus discovery. J. Virol. Methods 195, 194-204. doi: 10.1016/j.jviromet.2013.08.035

Head, S. R., Komori, H. K., LaMere, S. A., Whisenant, T., Van Nieuwerburgh, F., Salomon, D. R., et al. (2014). Library construction for next-generation sequencing: overviews and challenges. Biotechniques 56, 61-77. doi: $10.2144 / 000114133$

Junttila, N., Lévêque, N., Magnius, L. O., Kabue, J. P., Muyembe-Tamfum, J. J., Maslin, J., et al. (2015). Complete coding regions of the prototypes enterovirus $\mathrm{B} 93$ and C95: phylogenetic analyses of the P1 and P3 regions of EV-B and EV-C strains. J. Med. Virol. 87, 485-497. doi: 10.1002/jmv.24062

Knowles, N., Hovi, T., Hyypiä, T., King, A. M. Q., Lindberg, A. M., Pallansch, M., et al. (2012). "Picornaviridae," in Ninth Report of the International Committee on Taxonomy of Viruses, eds A. M. Q. King, M. J. Adams, E. B. Carstens, and E. J. Lefkowitz (Amsterdam: Elsevier Academic Press), 855-880.

Kyriakopoulou, Z., Pliaka, V., Amoutzias, G. D., and Markoulatos, P. (2015). Recombination among human non-polio enteroviruses: implications for epidemiology and evolution. Virus Genes 50, 177-188. doi: 10.1007/s11262014-1152-y

Monpoeho, S., Dehée, A., Mignotte, B., Schwartzbrod, L., Marechal, V., Nicolas, J. C., et al. (2000). Quantification of enterovirus RNA in sludge samples using single tube real-time RT-PCR. Biotechniques 29, 88-93. Available online at: http://www.biotechniques.com/BiotechniquesJournal/2000/July/ Quantification-of-Enterovirus-RNA-in-Sludge-Samples-Using-Single-TubeReal-Time-RT-PCR/biotechniques-44174.html 
Mount, D. W. (2007). Using the Basic Local Alignment Search Tool (BLAST). CSH Protoc. 2007:pdb top17. doi: 10.1101/pdb.top17

Nelson, C. W., and Hughes, A. L. (2015). Within-host nucleotide diversity of virus populations: insights from next-generation sequencing. Infect. Genet. Evol. 30, 1-7. doi: 10.1016/j.meegid.2014.11.026

Nix, W. A., Oberste, M. S., and Pallansch, M. A. (2006). Sensitive, seminested PCR amplification of VP1 sequences for direct identification of all enterovirus serotypes from original clinical specimens. J. Clin. Microbiol. 44, 2698-2704. doi: 10.1128/JCM.00542-06

Poptsova, M. S., Il'icheva, I. A., Nechipurenko, D. Y., Panchenko, L. A., Khodikov, M. V., Oparina, N. Y., et al. (2014). Non-random DNA fragmentation in next-generation sequencing. Sci. Rep. 4:4532. doi: 10.1038/ srep04532

Quiñones-Mateu, M. E., Avila, S., Reyes-Teran, G., and Martinez, M. A. (2014). Deep sequencing: becoming a critical tool in clinical virology. J. Clin. Virol. 61, 9-19. doi: 10.1016/j.jcv.2014.06.013

Rakoto-Andrianarivelo, M., Guillot, S., Iber, J., Balanant, J., Blondel, B., Riquet, F., et al. (2007). Co-circulation and evolution of polioviruses and species C enteroviruses in a district of Madagascar. PLoS Pathog. 3:e191. doi: 10.1371/journal.ppat.0030191

Rakoto-Andrianarivelo, M., Rousset, D., Razafindratsimandresy, R., Chevaliez, S., Guillot, S., Balanant, J., et al. (2005). High frequency of human enterovirus species C circulation in Madagascar. J. Clin. Microbiol. 43, 242-249. doi: 10.1128/JCM.43.1.242-249.2005

Rosseel, T., Van Borm, S., Vandenbussche, F., Hoffmann, B., van den Berg, T., Beer, M., et al. (2013). The origin of biased sequence depth in sequenceindependent nucleic acid amplification and optimization for efficient massive parallel sequencing. PLoS ONE 8:e76144. doi: 10.1371/journal.pone.0076144

Sadeuh-Mba, S. A., Bessaud, M., Massenet, D., Joffret, M. L., Endegue, M. C., Njouom, R., et al. (2013). High frequency and diversity of species C enteroviruses in cameroon and neighboring countries. J. Clin. Microbiol. 51, 759-770. doi: 10.1128/JCM.02119-12

Savolainen-Kopra, C., and Blomqvist, S. (2010). Mechanisms of genetic variation in polioviruses. Rev. Med. Virol. 20, 358-371. doi: 10.1002/rmv.663
Tapparel, C., Siegrist, F., Petty, T. J., and Kaiser, L. (2013). Picornavirus and enterovirus diversity with associated human diseases. Infect. Genet. Evol. 14, 282-293. doi: 10.1016/j.meegid.2012.10.016

Thomson, E., Ip, C. L., Badhan, A., Christiansen, M. T., Adamson, W., Ansari, M. A., et al. (2016). Comparison of next generation sequencing technologies for the comprehensive assessment of full-length hepatitis C viral genomes. J. Clin. Microbiol. doi: 10.1128/JCM.00330-16. [Epub ahead of print].

Tokarz, R., Haq, S., Sameroff, S., Howie, S. R., and Lipkin, W. I. (2013). Genomic analysis of coxsackieviruses A1, A19, A22, enteroviruses 113 and 104: viruses representing two clades with distinct tropism within enterovirus C. J. Gen. Virol. 94(Pt 9), 1995-2004. doi: 10.1099/vir.0.053462-0

van Dijk, E. L., Jaszczyszyn, Y., and Thermes, C. (2014). Library preparation methods for next-generation sequencing: tone down the bias. Exp. Cell Res. 322, 12-20. doi: 10.1016/j.yexcr.2014.01.008

Wright, C. F., Morelli, M. J., Thebaud, G., Knowles, N. J., Herzyk, P., Paton, D. J., et al. (2011). Beyond the consensus: dissecting within-host viral population diversity of foot-and-mouth disease virus by using next-generation genome sequencing. J. Virol. 85, 2266-2275. doi: 10.1128/JVI.01396-10

Zaidi, S. S., Asghar, H., Sharif, S., and Alam, M. M. (2016). Poliovirus laboratory based surveillance: an overview. Methods Mol. Biol. 1387, 11-18. doi: 10.1007/978-1-4939-3292-4_2

Conflict of Interest Statement: The authors declare that the research was conducted in the absence of any commercial or financial relationships that could be construed as a potential conflict of interest.

Copyright (๑) 2016 Bessaud, Sadeuh-Mba, Joffret, Razafindratsimandresy, Polston, Volle, Rakoto-Andrianarivelo, Blondel, Njouom and Delpeyroux. This is an openaccess article distributed under the terms of the Creative Commons Attribution License (CC BY). The use, distribution or reproduction in other forums is permitted, provided the original author(s) or licensor are credited and that the original publication in this journal is cited, in accordance with accepted academic practice. No use, distribution or reproduction is permitted which does not comply with these terms. 